\title{
Electroconductive Hydrogel Based on Functional Poly(Ethylenedioxy Thiophene)
}

Damia Mawad, ${ }^{*}, \dagger$, , I Arbel Artzy-Schnirman, ${ }^{\dagger, \mathbb{I}}$ Joanne Tonkin, ${ }^{\S}$ Jose Ramos, ${ }^{\dagger, \|}$ Sahika Inal, ${ }^{\perp}$ Muzamir M. Mahat, ${ }^{\dagger}$ Nadim Darwish, ${ }^{\#}$ Limor Zwi-Dantsis, ${ }^{\dagger}$ George G. Malliaras, ${ }^{\perp}$ J. Justin Gooding, ${ }^{\nabla}$ Antonio Lauto, ${ }^{\bigcirc}$ and Molly M. Stevens* ${ }^{\dagger} \dagger$

${ }^{\dagger}$ Department of Materials, Department of Bioengineering, Institute of Biomedical Engineering, Imperial College London, Prince Consort Road, London SW7 2AZ, United Kingdom

${ }^{\ddagger}$ School of Materials Science and Engineering, UNSW Australia, Sydney, New South Wales 2052, Australia

${ }^{\S}$ Faculty of Medicine, Imperial College London, London SW7 2AZ, United Kingdom

"POLYMAT, Bionanoparticles Group, University of the Basque Country UPV/EHU, Donostia-San Sebastián 20018, Spain

${ }^{\perp}$ Department of Bioelectronics, Ecole Nationale Superieure des Mines, CMP-EMSE, MOC, Gardanne 13541, France

\#Nanochemistry Research Institute, Department of Chemistry, Faculty of Science and Engineering, Curtin University, Perth, Western Australia 6102, Australia

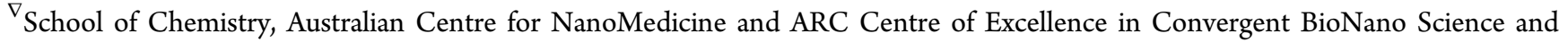
Technology, University of New South Wales, Sydney, New South Wales 2052, Australia

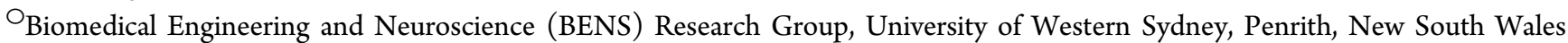
2751, Australia

\section{Supporting Information}

ABSTRACT: Poly(ethylene dioxythiophene) with functional pendant groups bearing double bonds is synthesized and employed for the fabrication of electroactive hydrogels with advantageous characteristics: covalently cross-linked porous 3D scaffolds with notable swelling ratio, appropriate mechanical properties, electroactivity in physiological conditions, and suitability for proliferation and differentiation of $\mathrm{C} 2 \mathrm{C} 12$ cells. This is a new approach for the fabrication of conductive engineered constructs.

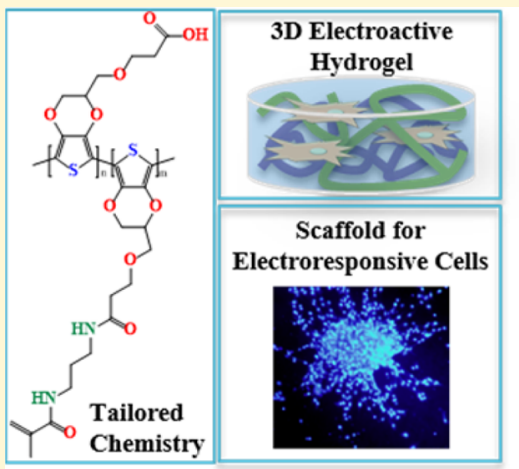

\section{INTRODUCTION}

Conducting polymers (CPs) have gained popularity in recent years as components of complex systems designed to electrically communicate with biological environments. ${ }^{1}$ Indeed, CPs provide new ways to communicate with cells, tissues, and other multicellular structures as they can avoid the mechanical mismatch of harder inorganic based materials and are also able to exhibit ionic in addition to electronic conductivity. $^{2}$ This is highlighted by the use of CPs as active components across a broad range of biotic/abiotic interfaces, with pioneering examples including the electronic plant, ${ }^{3}$ organic electrochemical transistor, ${ }^{4}$ neural cell membrane mimicking electrode, ${ }^{5}$ and multiplex immunoassay biosensor. ${ }^{6}$ Among the many forms they have been processed into, such as multilayered films, ${ }^{7,8}$ fibers, ${ }^{9}$ and sponges, ${ }^{10}$ CPs are being considered as a structural material for the fabrication of electroconductive hydrogels. ${ }^{11}$ In tissue engineering, mimicking the properties of the native extracellular matrix such as its hydrated nature is essential. In the case of electro-responsive organs such as the heart and nervous system, the engineered scaffold would also be required to be electrically conductive to reinstate the electronic functionality to the tissue. ${ }^{12}$ Nevertheless, the fabrication of electroconductive hydrogels based on CPs has proven challenging. CPs exhibit (i) high stiffness due to their inherently rigid backbone containing conjugated double bonds, ${ }^{13,14}$ (ii) a hydrophobic nature due to the aromatic rings in the backbone, and (iii) unwanted cross-links induced by $\pi-\pi$ stacking of the chains. ${ }^{15}$ In contrast, a 3D hydrogel network consists of cross-linked hydrophilic polymers with high water content, exhibits elastic behavior, and has porous internal

Received: March 31, 2016

Revised: July 25, 2016

Published: July 25, 2016 
structures. ${ }^{16,17}$ Here, we report a new fabrication approach for a functional poly(ethylene dioxythiophene) (PEDOT) based hydrogel that overcomes the aforementioned challenges. We chemically polymerized a PEDOT backbone functionalized with ionic pendant groups to synthesize a water-soluble processable conjugated polymer without compromising its electronic properties. Additionally, the side functionalities allowed postfunctionalization of the polymer to be tailored for desired scaffold requirements. Copolymerizing of the functional PEDOT with acrylic acid led to a 3D hydrogel with high water content, a range of mechanical properties that match that of a number of soft tissues, and electroactivity in physiological conditions. This is the first report of a conductive network built from covalently linked conjugated and hydrophilic polymers.

Initial approaches have shown that hydrogels could be made temporarily conductive either by fabricating hybrid hydrogels in which the CP is grown in a prefabricated network ${ }^{18,19}$ or as single component nonhybrid conductive hydrogels ${ }^{20,21}$ where the $\mathrm{CP}$ is the sole polymeric component; however, challenges remain in their application as components of bioelectronic devices. Hybrid hydrogels are comprised of CP chains that are physically or ionically entrapped within the conventional hydrogel matrix which limit their functionality in physiological conditions. As the hydrogel swells at physiological $\mathrm{pH}$, physically entrapped CP chains leach out, which triggers toxicity and causes a drop in electroactivity. ${ }^{22}$ Ionically bound CPs are neutralized at $\mathrm{pH}=7.4$ and eventually leach out of the network, which leads to the same detrimental drop in properties. $^{23}$ Nonhybrid conductive hydrogels are developed by self-assembly of the polymeric chains, ${ }^{10,24}$ chemical crosslinking, ${ }^{20}$ metal ions chelation, ${ }^{25}$ freeze-drying of $\mathrm{CP}$ dispersions, ${ }^{26}$ or employing a low molecular weight gelator (LMWG). ${ }^{27,28}$ The end product is a hydrogel network with enhanced electron transport between the conjugated chains due to the absence of insulating polymers from the matrix. However, the chemistry currently employed does not allow for the tuning of properties such as swelling or the mechanical characteristics of these scaffolds and is not easily amenable for tailoring to accommodate specific requirements in tissue engineering. In particular, the stability of hydrogels fabricated via self-assembly or LMWG in physiological conditions has never been tested. It could be envisioned that ionic strength will have an effect on the physicochemical properties of a network and its assembly. A new strategy is presented in this study to avoid the loss of the CP chains followed by a drop in the material's electronic properties.

An ideal precursor for a conductive hydrogel is a CP with the following properties: (i) water solubility to enhance swelling capacity, (ii) functional groups in the side chains that allow chemical cross-linking to eliminate polymer leaching following swelling in $\mathrm{pH} \mathrm{7.4,} \mathrm{and} \mathrm{(iii)} \mathrm{electrical} \mathrm{conductivity} \mathrm{in}$ physiological buffers. Here, we report a functional PEDOT with pendant carboxylic groups (PEDOT-COOH) that meets all these requirements: water solubility, functionalizable with demonstrated postmodification capabilities, and preserved electronic properties upon gelation. PEDOT with pendant carboxylic groups has been previously reported but via electropolymerization; ${ }^{29}$ this limits the scope of application since the polymer can only be used on electrode surfaces. Reports on chemically polymerized PEDOT have focused on alkylated, alkoxylated, or alkylsulfonate derivatives of EDOT. $^{30,31}$ However, these substituents do not offer the potential for the postfunctionalization of the polymer and thus limit their application in fabricating scaffolds. Furthermore, the functional carboxylic group ionizes at physiological $\mathrm{pH}$ (7.4) and will allow enhanced swelling of the developed scaffolds. We chose PEDOT due to its high stability in aqueous solutions ${ }^{32}$ and its demonstrated biocompatibility. ${ }^{33}$ By synthesizing for the first time a functional PEDOT bearing carboxylic groups via chemical polymerization, we obtained an electroactive polymer that could be cross-linked with hydrophilic polymers to form 3D scaffolds with retained electroactivity, high swelling ratios, and soft mechanical properties.

\section{EXPERIMENTAL SECTION}

Materials. Hydroxymethyl EDOT (EDOT-OH) was purchased from Molekula Ltd., UK. All other chemicals were purchased from Sigma UK and used without any further purification.

Synthesis of PEDOT-COOH. EDOT-COOCH ${ }_{3}$ EDOT-OH $(1.0 \mathrm{~g})$ was dissolved in anhydrous terahydrofuran (THF) $(25 \mathrm{~mL})$ under the flow of $\mathrm{N}_{2}$, and the solution was cooled down by immersing the $\mathrm{RB}$ flask in an ice bath. Potassium iodide $(0.19 \mathrm{~g})$ and a $\mathrm{NaH}$ suspension $(0.278 \mathrm{~g})$ were added, and the solution was left stirring for $30 \mathrm{~min}$. Methyl-3-bromoproprionate $(0.76 \mathrm{~mL})$ was then added dropwise, and the reaction was left stirring under $\mathrm{N}_{2}$ for $48 \mathrm{~h}$. Deionized water (DI$\mathrm{H}_{2} \mathrm{O}$ ) was added to quench the reaction, and THF was removed under reduced pressure. The aqueous mixture was extracted with ethyl acetate, dried over $\mathrm{MgSO}_{4}$, filtered, and concentrated under reduced pressure. The crude product was purified by flash chromatography (hexane/ethyl acetate $=5: 1$ ) to give a clear viscous liquid. ${ }^{1} \mathrm{H}$ NMR $\left(400 \mathrm{MHz}, \mathrm{CDCl}_{3}\right): \delta 6.32(2 \mathrm{H}, \mathrm{s}), 4.26-4.33(1 \mathrm{H}, \mathrm{m}), 4.22(1 \mathrm{H}, \mathrm{dd}$, $J=11.6,2.3 \mathrm{~Hz}), 4.03(1 \mathrm{H}, \mathrm{dd}, J=11.6,7.5 \mathrm{~Hz}), 3.79(2 \mathrm{H}, \mathrm{t}, J=6.3$ $\mathrm{Hz}), 3.67(3 \mathrm{H}, \mathrm{s}), 3.62-3.76(2 \mathrm{H}, \mathrm{m}), 2.62(2 \mathrm{H}, \mathrm{t}, J=6.3 \mathrm{~Hz}$ ) (Figure S1).

PEDOT-COOH. The functional PEDOT polymer was synthesized by chemical oxidation using $\mathrm{FeCl}_{3}$ as the oxidant. In a 2-neck $\mathrm{RB}$ flask, $\mathrm{FeCl}_{3}(1.45 \mathrm{~g}, 8.94 \mathrm{mmol})$ and anhydrous chloroform $(32 \mathrm{~mL})$ were mixed under the flow of $\mathrm{N}_{2}$. EDOT-COOCH${ }_{3}(0.64 \mathrm{~g}, 2.48 \mathrm{mmol})$ was dissolved in anhydrous chloroform $(6 \mathrm{~mL})$ and added dropwise to the oxidant mixture and left stirring for $24 \mathrm{~h}$ at room temperature. The mixture was poured in methanol $(\sim 500 \mathrm{~mL})$ and centrifuged to collect the solid. The precipitate was washed thoroughly with methanol and then with water until the solvent was clear. To remove the methyl group on the side chain, the polymer was treated with $2 \mathrm{M} \mathrm{NaOH}$ (50 $\mathrm{mL}$ ) for 1 day at room temperature and then filtered to remove the insoluble parts. The filtrate was purple in color and treated with $10 \%$ $\mathrm{HCl}$ to precipitate the polymer. The polymer was washed with water and dried under vacuum to obtain a black solid $(\sim 100 \mathrm{mg})$.

Characterization of PEDOT-COOH. Solid State NMR. The chemical structure of PEDOT-COOH was confirmed using a Bruker Avance 111 solid state NMR spectrometer operating at $75 \mathrm{MHz}$ for ${ }^{13} \mathrm{C}$ and 299.75 for ${ }^{1} \mathrm{H}$ and fitted with a $4 \mathrm{~mm}$ CPMAS probe. Sample $(\sim 60 \mathrm{mg})$ was packed into a $4 \mathrm{~mm}$ zirconia oxide rotor. Spectra were acquired with MAS of $12 \mathrm{kHz}$, using a spin echo sequence allowing for direct ${ }^{13} \mathrm{C}$ detection with high power ${ }^{1} \mathrm{H}$ decoupling with spinal 64 sequence at $62 \mathrm{kHz}$. The relaxation delay was set to $20 \mathrm{~s}$, and total instrument time was $\sim 13 \mathrm{~h}$. Cross-polarization failed to give any signal.

${ }^{1} H$ NMR. PEDOT-COOH $(4.9 \mathrm{mg})$ was dissolved in DMSO- $d_{6}$ $(770.93 \mathrm{mg})$, and its NMR spectroscopy was recorded using a Bruker Avance 111, HD 600 with $5 \mathrm{~mm}$ TCI cryoprobe. NMR spectra were processed using the Bruker TOPSPIN 3.0 software.

Gel Permeation Chromatography (GPC). GPC was performed using phosphate buffered saline (PBS, $0.1 \mathrm{M}$ ) (flow rate: $0.5 \mathrm{~mL}$ $\left.\min ^{-1}\right)$. A Shimadzu modular system was employed comprising a DGU-20A3 solvent degasser, an LC-20AT pump, a CTO-20A column oven operated at $35{ }^{\circ} \mathrm{C}$, and a SPD-M20A diode array UV-vis detector $(200-800 \mathrm{~nm})$. The system was equipped with an Ultrahydrogel 250 column $(6 \mu \mathrm{m}, 7.8 \times 300 \mathrm{~mm})$ (Waters, WAT01152). Calibration was achieved using sodium poly- 
(styrenesulfonate) standards (Polymer Laboratories LTD, UK) ranging from 1400 to $32000 \mathrm{~g} \mathrm{~mol}^{-1}$.

Chemical Doping. Drop-casted films of PEDOT-COOH were chemically doped by treating the surface with $0.1 \mathrm{M} \mathrm{HClO}_{4}$ aqueous solution for $24 \mathrm{~h}$. Excess dopant was wiped gently off the surface, and the films were rinsed for $30 \mathrm{~s}$ with $\mathrm{DI}-\mathrm{H}_{2} \mathrm{O}$, dried with $\mathrm{N}_{2}$, and stored in the dark until use.

UV-vis Spectroscopy. Absorbance spectra were recorded using a PerkinElmer Lambda $25 \mathrm{UV}$-vis spectrophotometer. The spectra of PEDOT-COOH dissolved in water, $1 \mathrm{M} \mathrm{NaOH}$, and dimethyl sulfoxide (DMSO) as well as that of drop-casted films (doped and undoped) were recorded. The optical band gap, $E_{\text {gopt }}$ was estimated from the UV-vis spectra of undoped PEDOT-COOH film. The linear portion of the curve was extrapolated to the wavelength axis, and the intercept was determined to be the absorption edge wavelength, $\lambda_{\mathrm{ab}}$. $E_{\text {g,opt }}$ was calculated as follows: ${ }^{34,35}$

$$
E_{\mathrm{g}, \mathrm{opt}}=h c / \lambda_{\mathrm{ab}}
$$

where $h$ is the Planck constant and $c$ is the speed of light.

Spectroelectrochemistry. In situ spectroelectrochemical measurements were conducted on undoped PEDOT-COOH films prepared by drop casting on ITO slides $(1 \times 4 \mathrm{~cm})$. The electrolyte solution was $0.1 \mathrm{M}$ tetrabutylammonium tetrafluoroborate (TBAB) in acetonitrile. The reference electrode used was a $\mathrm{Ag}$ wire with $\mathrm{Pt}$ wire as auxiliary. The applied voltages on the ITO modified electrode were rescaled vs $\mathrm{Ag} / \mathrm{AgCl}$ by using ferrocene. The controlled-potential measurements were carried out with an $\mathrm{EDAQ}$ system controlled by EChem software.

Electrochemistry. Cyclic voltammetry (CV) measurements were recorded using CHI660D Instrument. The counter electrode was a platinum mesh, and the reference electrode was $\mathrm{Ag} / \mathrm{AgCl}$ in saturated $\mathrm{KCl}$ aqueous solution ( $\mathrm{CHI}$ Instruments). The working electrode was ITO coated glass (Delta Technologies Limited) on which the PEDOT-COOH polymer was drop casted. Electrochemical measurements were performed at different scan rates using $0.1 \mathrm{M} \mathrm{TBAB}$ in acetonitrile as the electrolyte solution. The onset of the oxidation potential, $E_{\mathrm{ox}}$, was determined from the $\mathrm{CV}$. The HOMO-LUMO levels were determined as follows: ${ }^{36}$

$$
\begin{aligned}
& \text { HOMO }=-\left(E_{\text {ox }}+4.80\right) \mathrm{eV} \\
& \mathrm{LUMO}=\left(\mathrm{HOMO}+E_{\mathrm{g}, \mathrm{opt}}\right) \mathrm{eV}
\end{aligned}
$$

Hydrogel Fabrication. Functional PEDOT (f-PEDOT). To introduce a double bond in the side chain of PEDOT-COOH, the polymer was reacted with $\mathrm{N}$-(3-aminopropyl) methacrylamide hydrochloride (APMA). PEDOT-COOH (0.355 g) was dissolved in DMSO $(30 \mathrm{~mL})$ at $60{ }^{\circ} \mathrm{C}$ and then cooled to room temperature. APMA $(0.117 \mathrm{~g})$ and $\mathrm{N}$-(3-(dimethylamino)propyl)- $\mathrm{N}^{\prime}$-ethylcarbodiimide hydrochloride (EDC) (0.249 g) were added, and the reaction was left to stir for $24 \mathrm{~h}$. To wash out all the byproducts and unreacted monomer (APMA), the solution was poured into excess water, which led to the precipitation of a black solid. The black solid was collected by centrifugation, washed repeatedly with water, and dried under vacuum to give $\mathrm{f}$-PEDOT in powder form.

${ }^{1} H$ NMR. f-PEDOT $(5.0 \mathrm{mg})$ was dissolved in DMSO- $d_{6}(768.25$ $\mathrm{mg}$ ), and its NMR spectroscopy was recorded as described above. The degree of substitution of APMA was quantified using an external reference (trioxane $(10.5 \mathrm{mg})$ in DMSO- $d_{6}(771.65 \mathrm{mg})$ ) and using Topspin Eretic Software.

Elemental Analysis. Elemental analysis was performed at Campbell Microanalytical Laboratory, University of Otago, New Zealand. Samples were precisely weighed on a Mettler UMT2 microbalance into lightweight tin capsules and dropped into a combustion tube of the elemental analyzer (Carlo Erba EA 1108) through which a constant stream of helium is maintained. Total carbon, hydrogen, nitrogen, and sulfur were determined for both PEDOT-COONa and fPEDOT.

f-PEDOT Hydrogel. To fabricate a cross-linked functional PEDOT hydrogel, f-PEDOT was copolymerized with acrylic acid (AA) in the presence of poly(ethylene glycol) diacrylate (PEG-DA, average molecular number $\left.\left(M_{\mathrm{n}}\right)=700\right)$ as a cross-linker and 2,2'-azobis(2methyl-proprionitrile) (AIBN) as the initiator. In a typical procedure, f-PEDOT $(5 \mathrm{mg})$ was dissolved in DMSO $(1 \mathrm{~mL})$ at $60{ }^{\circ} \mathrm{C}$ to a final concentration of $0.5 \mathrm{wt} \%$ polymer. The solution was then cooled to room temperature. AA $(95.2 \mu \mathrm{L})$, PEG-DA $(10 \mu \mathrm{L})$, and AIBN (2 $\mathrm{mg}$ ) were added to the polymer solution, and it was vortexed and left at $60{ }^{\circ} \mathrm{C}$ for $24 \mathrm{~h}$. Gelation of the solutions was confirmed by the test tube inverting method. The vials containing the mixture were inverted, and gelation was deemed to have occurred when no flow of liquid was observed. The hydrogels were then removed from the vials, washed with excess $10 \%(\mathrm{w} / \mathrm{v}) \mathrm{HCl}$ for 1 day, and then soaked in excess DI$\mathrm{H}_{2} \mathrm{O}$ for 3 days. The hydrogels were then freeze-dried and stored until use. Poly (acrylic acid) (PAA) hydrogels were fabricated according to the same procedure but without adding f-PEDOT in the solution.

Characterization of the Physical Properties of $f$-PEDOT Hydrogel. Swelling Studies. The dry weights of freeze-dried hydrogels were recorded $\left(m_{\mathrm{d}}\right)$ before incubating the samples in PBS $\left(0.1 \mathrm{M}, \mathrm{pH}=7.3,37^{\circ} \mathrm{C}\right)$ and then leaving them to swell until equilibrium was reached. At predetermined time points, the samples were removed and gently blotted, and their swollen weight $\left(m_{s}\right)$ was recorded. The percentage-swelling ratio was calculated according to the following equation:

$$
\% \text { swelling ratio }=\frac{m_{\mathrm{s}}-m_{\mathrm{d}}}{m_{\mathrm{d}}} \times 100
$$

A total of 9 specimens were measured at each time point.

Mechanical Properties. The compression modulus of cylindrical hydrogels was measured at room temperature using a Bose Instrument equipped with a $3 \mathrm{~N}$ load cell. Samples were removed from the swelling media and blotted dry to get rid of excess water. The thickness and radius $(r)$ of each sample was measured before running the test (listed in Table S1). The samples were placed between parallel plates and compressed at $0.1 \% / \mathrm{s}$ strain rate. The stress was calculated according to

$$
\text { stress }=\frac{\text { load }}{\pi \times r^{2}}
$$

where $r$ is the initial unloaded radius. The strain under compression was defined as the change in the thickness relative to the thickness of the freestanding specimen. The compression modulus was calculated from the slope of the linear region of the stress-strain plot at less than $15 \%$ deformation. Results were reported for hydrogels in two different states: swollen in PBS $(n=5)$ and $\mathrm{HClO}_{4}$-doped hydrogels $(n=4)$.

Characterization of the Electronic Properties of $f$-PEDOT Hydrogel. Chemical Doping of $f-P E D O T$ Hydrogel. Hydrogels were soaked in $0.1 \mathrm{M} \mathrm{HClO}_{4}$ aqueous solution for $24 \mathrm{~h}$. This was followed by rinsing with water and freeze-drying the samples.

Electrochemistry. Freeze-dried hydrogels (undoped and doped) were anchored on gold mylar surfaces and soaked in PBS for 10-15 min to allow for water uptake. CVs were then recorded as described above but using PBS ( $\mathrm{pH}=7.4)$ as the electrolyte.

Impedance. The electrochemical impedance (EIS) spectra of PAA (control) and f-PEDOT hydrogels were measured using Solartron SI 1287 electrochemical interface coupled with an SI 1260 frequency response analyzer (Solectron Analytical, Hampshire, England) from 10 $\mathrm{kHz}$ to $1 \mathrm{~Hz}$ with an applied AC potential of $10 \mathrm{mV}$ versus the reference electrode potential. Before recording the measurements, hydrogels were anchored on gold mylar and incubated in PBS for $24 \mathrm{~h}$ to ensure swelling to equilibrium. $\mathrm{Ag} / \mathrm{AgCl}$ and $\mathrm{Pt}$ electrodes were used as the reference and counter electrodes, respectively. The applied DC voltage was $290 \mathrm{mV}$, and the electrolyte solution was PBS (0.1 $\mathrm{M})$.

In Vitro Cell Studies. Cell Lines and Reagents. All reagents were purchased from Life Technologies (UK) unless otherwise stated. Mouse $\mathrm{C} 3 \mathrm{H}$ muscle myoblast line $(\mathrm{C} 2 \mathrm{C} 12)$ was purchased from ATCC. Horse serum was purchased from Sigma. Cells were maintained in Dulbecco's modified Eagle's medium (DMEM) supplemented with $20 \%(\mathrm{v} / \mathrm{v})$ fetal bovine serum (FBS) and antibiotics (100 units $/ \mathrm{mL}$ penicillin and $100 \mu \mathrm{g} / \mathrm{mL}$ streptomycin), under standard mammalian cell culture conditions. The high 
concentration of FBS provides a rich source of mitogenic growth factors required for optimal cell growth and proliferation while the addition of antibiotics prevents the growth of potential bacterial contaminants. Media was replaced every other day.

Cell Culture. Hydrogels were transferred into 24 well plates and sterilized by cell culture-grade UV light irradiation for $1 \mathrm{~h}$. The samples were then soaked in DI- $\mathrm{H}_{2} \mathrm{O}$ overnight prior to cell seeding in order to wash excess acid from the doped samples. The next day, to meet the required environment for cell culture, water was removed from the wells and replaced with media for $1 \mathrm{~h}$ which was then removed before seeding the cells. $\mathrm{C} 2 \mathrm{C} 12$ cells were detached from the culture plates using trypsin, followed by centrifugation $(6000 \mathrm{~g}, 5 \mathrm{~min})$. The cell pellet was resuspended in fresh media. Cells $\left(1.5 \times 10^{6}\right.$ cells in $30 \mu \mathrm{L}$ ) were dispensed on the surface of the hydrogels and left for 1 $\mathrm{h}$ to allow for adhesion. Adherence of $\mathrm{C} 2 \mathrm{C} 12 \mathrm{~s}$ to the surface is necessary for the cells to remain viable and for all cell function (cell division, migration, and maturation). The wells were then filled with growth media and allowed to proliferate for 3 days. Cells were then washed to remove traces of the growth medium. Although the greatest care was taken, some cells could have been dislodged during this process. The cells attached to the hydrogels were then placed in a differentiating media (DMEM with $2 \%$ (v/v) horse serum, 100 units/ $\mathrm{mL}$ penicillin, and $100 \mu \mathrm{g} / \mathrm{mL}$ streptomycin antibiotics) and incubated for 5 days. Horse serum was included in the differentiation medium to provide a minimal level of nutrients which are needed to keep the cells healthy and viable. At various time points, differentiation media was removed and the cells/hydrogels were washed in PBS before being submerged in freshly prepared fixation buffer $(4 \%(\mathrm{v} / \mathrm{v})$ paraformaldehyde in PBS) for $10 \mathrm{~min}$ at room temperature. Excess fixative was washed away with PBS.

Immunostaining. Fixed hydrogels were cut into $250 \mu \mathrm{m}$ crosssectional and longitudinal slices using a high precision vibrating microtome $(7000 \mathrm{smz}$, Campden Instruments Ltd., UK). The slices were blocked in 5\% (v/v) FBS in PBS for $2 \mathrm{~h}$ and then incubated overnight at $4{ }^{\circ} \mathrm{C}$ with primary antibodies that specifically bind to the proteins Ki67 and desmin (Abcam; ab8470 and ab15580) or myosin heavy chain IIb (MHC IIb, clone MF20; RnD systems). Unbound primary antibodies were removed by $3 \times 20$ min washes in PBS. To visualize the bound antibodies, the cells were incubated in secondary antibodies labeled with fluorophores (goat antimouse Alexa 488 and goat antirabbit Alexa 488; Invitrogen). For some experiments, cells/ hydrogels were also incubated in Alexa Fluor 594-phalloidin (Invitrogen). Phalloidin is a peptide that binds to actin in cells and allows visualization of cell shape. DAPI was used to label nuclei. Confocal microscopy was performed using a Zeiss LSM-780 inverted microscope.

\section{RESULTS AND DISCUSSION}

We achieved the synthesis of PEDOT-COOH (Scheme 1A) by oxidation polymerization of EDOT- $\mathrm{COOCH}_{3}$ (Figure S1) followed by deprotection in $\mathrm{NaOH}$; this yielded a polymer with carboxylic groups in the backbone (Figure S2A). The chemical structure of the polymer was also confirmed by solution NMR (broadened peaks, Figure S2B) and elemental analysis (Table S2). The resultant polymer had a weight-average molecular weight $\left(M_{\mathrm{w}}\right)$ of $10102 \mathrm{~g} \cdot \mathrm{mole}^{-1}$ and a polydispersity of 2.49 as determined by GPC. The polymer was soluble in DMSO ( $1 \mathrm{wt}$ $\%)$, water $(\sim 0.6 \mathrm{wt} \%)$, and basic aqueous $\mathrm{pH}$ solutions (10 wt $\%$ at $\mathrm{pH} 12)$. This conferred solubility provides an advantage over the more commonly used form of PEDOT, which is a dispersion in water stabilized with high concentrations of poly(styrenesulfonate) (PSS). Figures $1 \mathrm{~A}$ and S3 show the absorption spectra of PEDOT-COOH film and solutions, respectively. A broad absorption band, $\lambda_{\max } \approx 519 \mathrm{~nm}$ for the films and $480 \mathrm{~nm}$ for solutions, was detected corresponding to the $\pi-\pi^{*}$ transition in the conjugated backbone. ${ }^{32}$ The optical band gap $\left(E_{\text {g,opt }}\right)$ determined from the film spectra was $1.70 \pm$
Scheme 1. (A) Synthesis of PEDOT-COOH Polymer; (B) Fabrication of f-PEDOT Hydrogel

A)

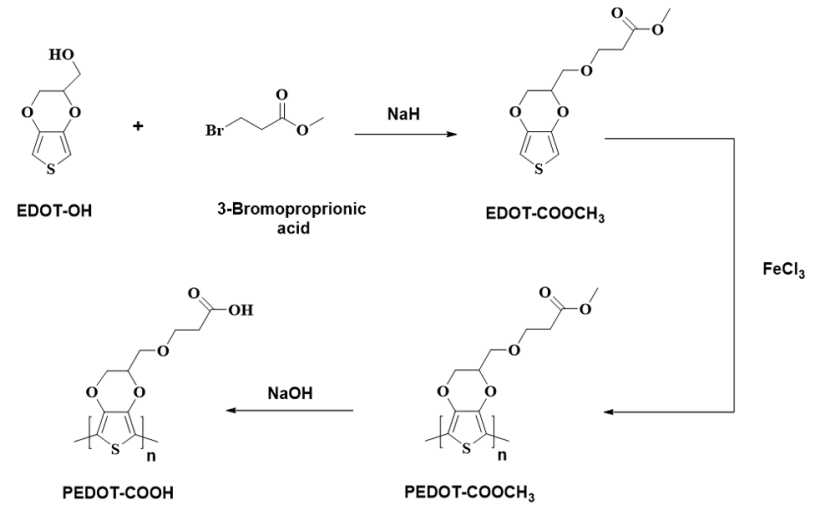

B)
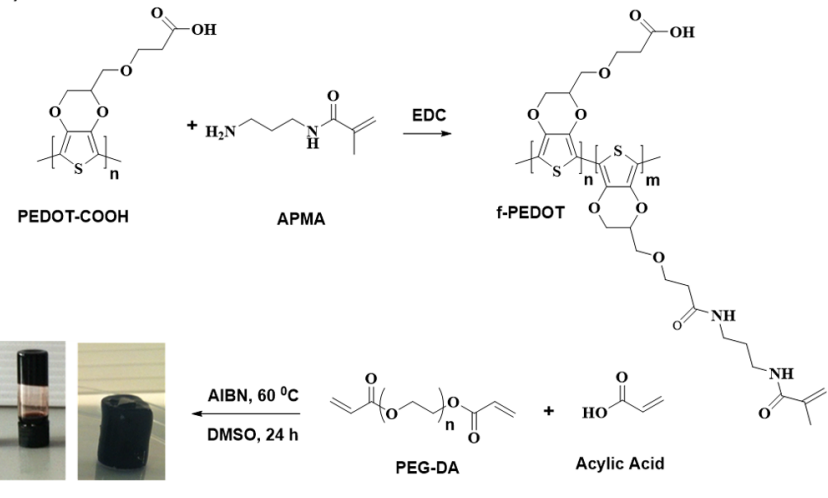

$0.09 \mathrm{eV}$. This value is in the range $(1.5-2.0 \mathrm{eV})$ of other chemically synthesized derivatives of PEDOT, ${ }^{32,34,37}$ which indicated that $\mathrm{EDOT}-\mathrm{COOCH}_{3}$ polymerization proceeded efficiently to produce a functional PEDOT polymer with sufficient conjugation length.

We conducted in situ spectroelectrochemistry to elucidate the electronic properties of the modified PEDOT (Figure 1A). In the absence of a bias, the film exhibited a broad peak at $~ 513$ $\mathrm{nm}$, which suggested that the film was in the anticipated reduced state following treatment in $\mathrm{NaOH}$. As the potential exceeded $0 \mathrm{~V}$, the intensity of the peak decreased along with the appearance of a new absorption feature in the near-infrared region $(>800 \mathrm{~nm})$, which is representative of polaronic states. This suggested a substantial change in the oxidation state of the PEDOT-COOH film from reduced to oxidized as the positive bias was applied. Chemical treatment of the films with $\mathrm{HClO}_{4}$ caused a similar switch between the reduced (undoped) and oxidized form (doped) (Figure 1B). Hence, it could be inferred that the PEDOT-COOH is a conjugated polymer with intrinsic electroactivity.

To further confirm the conjugated nature of the polymer, we examined the electrochemical behavior of the modified PEDOT in tetrabutylammonium tetrafluoroborate (TBAB) electrolyte solution (Figure 1C). The film exhibited good redox activity with a broad anodic $(0.314-0.735 \mathrm{~V})$ and a broad cathodic peak $(0.32-0.19 \mathrm{~V})$. This indicated that the electron transfer ability of the chemically polymerized PEDOT was not hindered by the carboxylic group in the side chain. A linear relation was also established between the peak current and the potential scan rate (Figure S4), which denoted that the electron transfer process was not diffusion limited ${ }^{38,39}$ and that the PEDOT film 

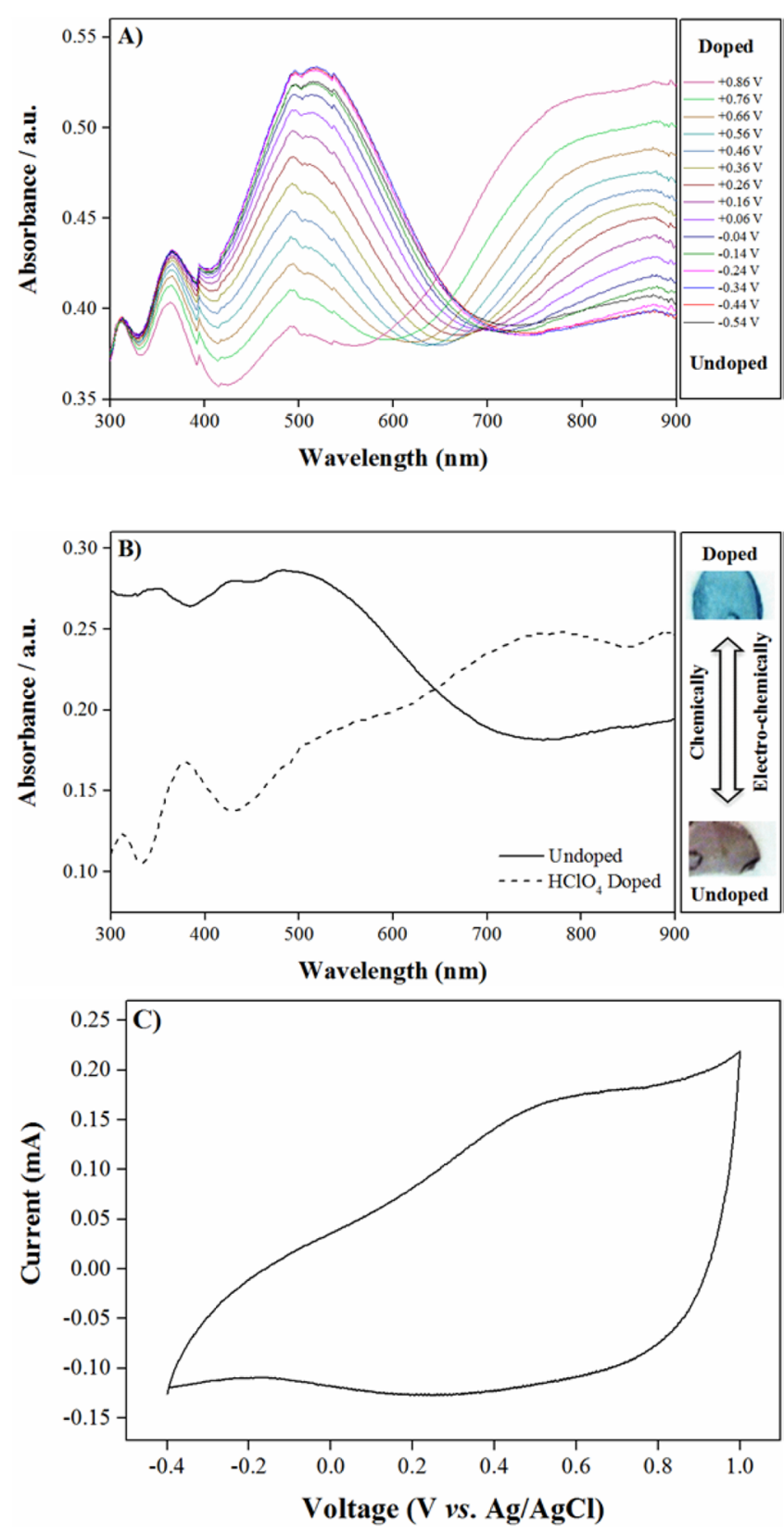

Figure 1. Monitoring the electronic properties of the PEDOT-COOH polymer. (A) Spectroelectrochemistry of a PEDOT-COOH film dropcasted on ITO-coated glass as a function of applied potential between -0.54 and $0.86 \mathrm{~V}$. (B) UV-vis absorption spectra of reduced (-) and $\mathrm{HClO}_{4}$ doped (- - ) PEDOT-COOH films. (C) Cyclic voltammogram $\left(25 \mathrm{mV} \cdot \mathrm{s}^{-1}\right)$ of PEDOT-COOH film drop-casted on ITO-coated glass in $\mathrm{TBAB}$ acetonitrile solution $(0.1 \mathrm{M})$.

was in intimate contact with the ITO electrode. From the experimental results of cyclic voltammetry $(\mathrm{CV})$ and $\mathrm{UV}$ spectrophotometry, the calculated values of the HOMO and LUMO of PEDOT-COOH were -5.28 and $-3.51 \mathrm{eV}$, respectively, which was comparable to other reported values for PEDOT polymers. ${ }^{34,37}$

Introducing a functional group in the side chain of PEDOT enabled postfunctionalization of the polymer. We reacted PEDOT-COOH with $\mathrm{N}$-(3-aminopropyl) methacrylamide hydrochloride (APMA) to introduce a double bond in the chain (f-PEDOT) (Figures S5 and S6A). Using an external reference (Figure S6B), the degree of substitution was determined to be $\sim 20 \mathrm{wt} \%$. This was confirmed by elemental analysis that showed the presence of nitrogen in f-PEDOT as opposed to PEDOT-COOH (Table S2) and a degree of substitution approximated at $\sim 16 \mathrm{wt} \%$. f-PEDOT was then polymerized for $24 \mathrm{~h}$ with acrylic acid (AA $)^{40}$ and poly(ethylene glycol) diacrylate (PEG-DA) to fabricate a 3D crosslinked gel comprised of poly(acrylic acid) (PAA) chains covalently linked to f-PEDOT via the PEG-DA (Scheme 1B). Each of the three components works synergistically to produce a conductive 3D hydrogel, namely: PAA is a polyelectrolyte that ionizes in physiological $\mathrm{pH}$ and causes water uptake; PEGDA is a cross-linker that covalently binds the polymeric chains to produce the $3 \mathrm{D}$ structure; PEDOT is a conjugated polymer that introduces redox and electronic activity into the scaffold.

The gel was removed from the glass vial and sectioned into specimens $5 \mathrm{~mm}$ in thickness. The gel is dark in color (Scheme 1B). While this could be a limiting factor for external bioapplications, many of the electroresponsive tissues that could benefit from the conductive gel are internal organs such as the heart, spinal cord, or the brain. The specimens were washed repeatedly with $10 \% \mathrm{HCl}$, which ensured the exchange of DMSO with acidic water without causing uncontrolled swelling of the gels if soaked immediately in water. The specimens were then transferred into excess DI- $\mathrm{H}_{2} \mathrm{O}$ and left to soak for $48 \mathrm{~h}$. Of significance here is that the chemically produced PEDOT can be functionalized with a plethora of compounds other than APMA or copolymerized with other monomers or polymers bearing double bonds.

Considering the hydrophobic nature of the f-PEDOT backbone due to the presence of aromatic rings, it was important to investigate whether the $\mathrm{CP}$ in the network hindered the swelling behavior of the hydrogel. Freeze-dried specimens were incubated in PBS $\left(\mathrm{pH}=7.4\right.$ at $\left.37^{\circ} \mathrm{C}\right)$ and weighed at different times. Hydrogels were found to exhibit a remarkable water uptake after $48 \mathrm{~h}(\sim 5000 \%)$, which was comparable to the swelling ratio of other PAA copolymer hydrogels (Figure 2A). ${ }^{41}$ The swelling of the hydrogels was due to the ionization at $\mathrm{pH}=7.4$ of the pendant carboxylic groups present on both polymers (PAA and f-PEDOT). As the proton of the carboxylic groups dissociates in $\mathrm{pH}=7.4$, electrostatic repulsion between the polymer chains caused the network to uptake water. Compared to previously reported conductive hydrogels, ${ }^{12,18,22}$ our f-PEDOT hydrogel displayed significantly higher ratio of swelling, which is an attractive property for applications in tissue engineering and drug delivery. With their high water content, these conductive hydrogels present tissue engineering advantages for soft tissue. Strong water uptake promises for efficient mass transfer, which is advantageous for applications of the hydrogel in cell proliferation and tissue formation.

It is well established that the stiffness of any scaffold dictates the cell-biomaterial interaction. ${ }^{42}$ Since the conductive hydrogel is designed to function as a scaffold for cell seeding and attachment, we characterized its mechanical properties. Additionally, the redox state of the polymer (doped versus undoped) might alter the mechanical properties of the network; hence, we measured the compressive moduli of both doped and undoped scaffolds (Figure 2B). The Young modulus of PBS treated samples was $8.2 \pm 3.1 \mathrm{kPa}$, respectively. In contrast, doped hydrogels displayed an increased stiffness $(45.14 \pm 18.78$ $\mathrm{kPa}, p<0.005$, Tukey's multiple comparison test, one-way ANOVA). Monitoring the swelling of the samples in the dopant solution $\left(0.1 \mathrm{M} \mathrm{HClO}_{4}\right)$ revealed minimal water uptake 

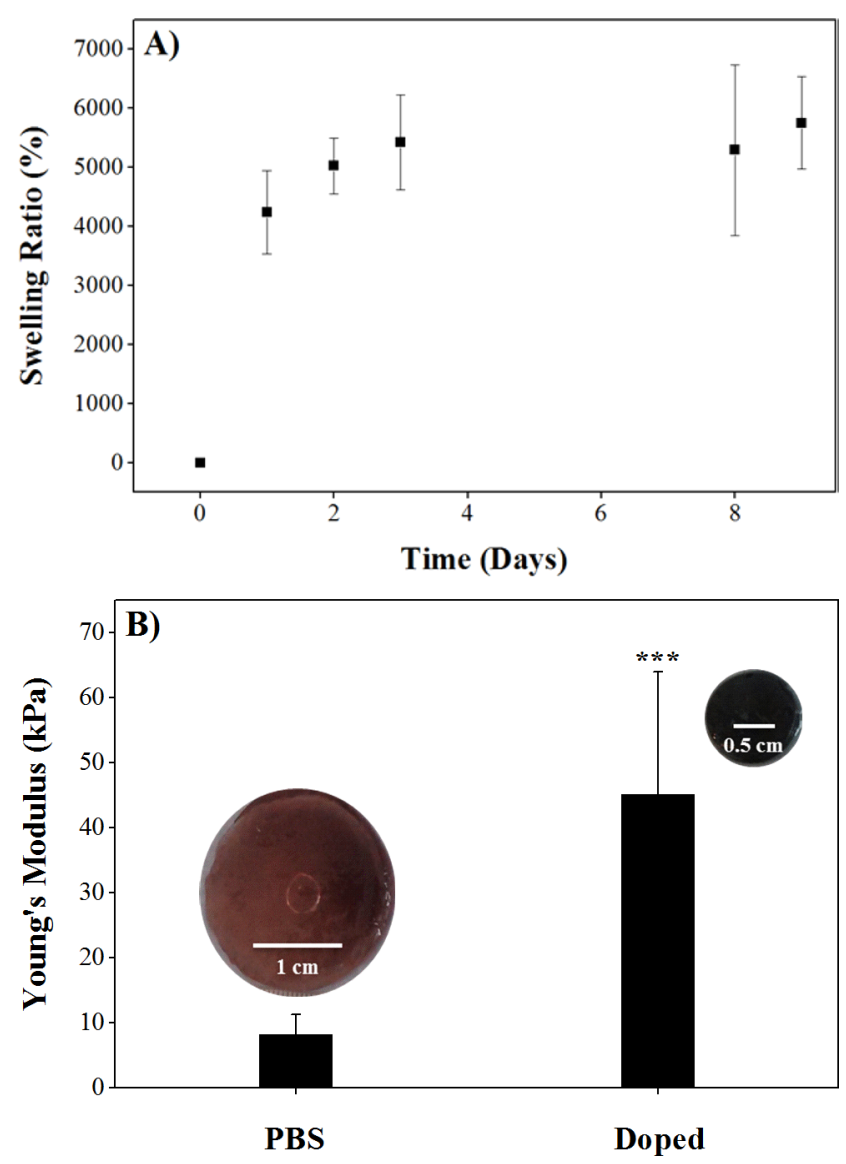

Figure 2. Monitoring the physical properties of the f-PEDOT hydrogel. (A) Percentage swelling ratio of undoped hydrogels. (B) Young's modulus of hydrogel networks swollen in different media $(p<$ $0.005)$.

compared to those in PBS (Figure S7). In a low $\mathrm{pH}$ media, the carboxylic groups are protonated and the polymeric chains come into closer vicinity, which could lead to secondary bonds between the aromatic rings, and in turn increases the mechanical strength of the network.

Having established that the f-PEDOT hydrogel displayed desirable physical characteristics of a network applicable to tissue engineering (high swelling ratios and mechanical integrity), we characterized its electronic properties to examine whether f-PEDOT retained its electroactivity in the presence of the insulators PAA and PEG-DA. Redox activity of the scaffold is important to show that the material being designed is capable of providing an electrical stimulus, which will ultimately allow for direct stimulation of cells and the transduction of biological signals to be promising. Figure 3A shows the electrochemistry of the f-PEDOT hydrogel in PBS as an electrolyte. The CVs exhibited characteristic features comparable to those of the PEDOT-COOH polymer (Figure 1C); broad anodic and reduction peaks at $\sim 0.48$ and $\sim 0.35 \mathrm{~V}$, respectively, with a halfwave potential, $E_{1 / 2}$, of $0.415 \mathrm{~V}$, which indicated that a clear effective charge transport is occurring in the f-PEDOT hydrogel despite the swollen state and the presence of insulating polymers (PAA and PEG) in the network. Furthermore, hydrogels subject to repeated cycles exhibited a stable electrochemical response (Figure S8).

After doping the hydrogels with $0.1 \mathrm{M} \mathrm{HClO}_{4}$, we observed a shift in the $E_{1 / 2}$ to lower potentials $\left(E_{1 / 2}=0.29 \mathrm{~V}\right.$; anodic peak
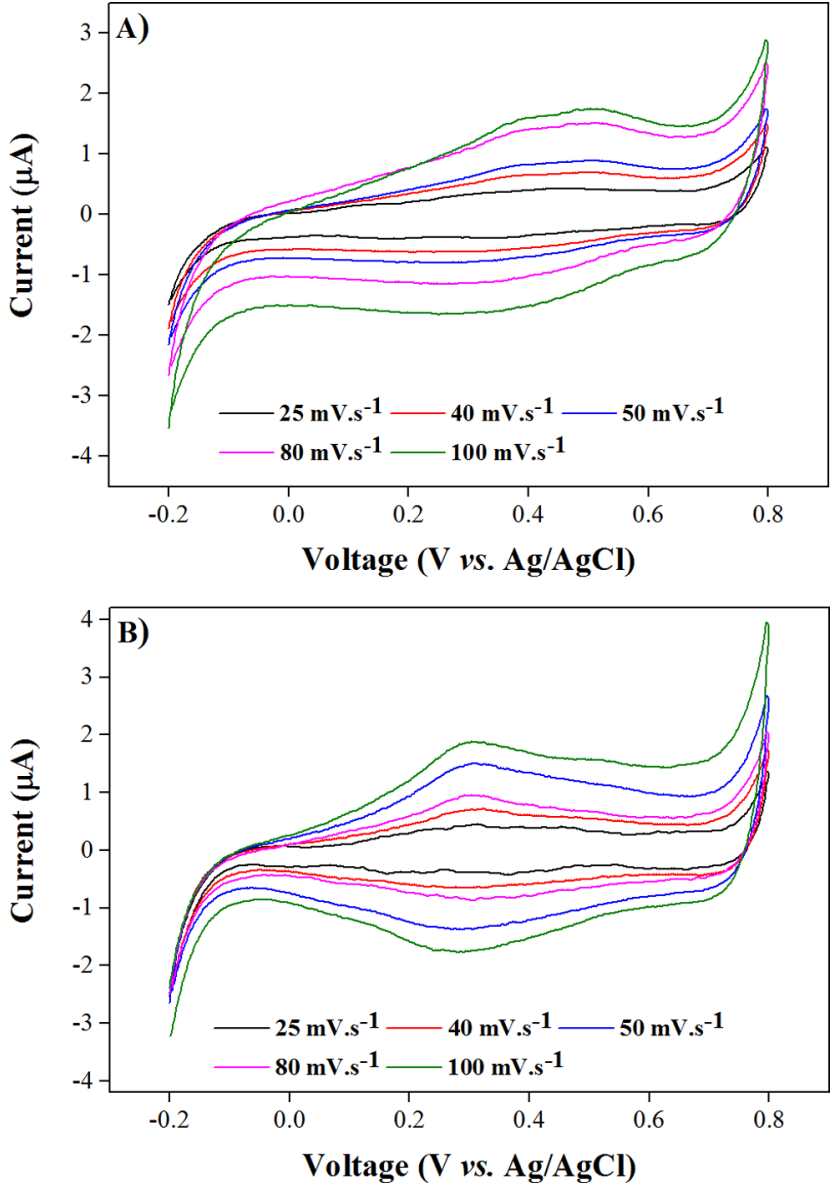

Figure 3. Cyclic voltammograms of (A) undoped and (B) doped hydrogels in $0.1 \mathrm{M}$ PBS conducted at different potential scan rates.

at $0.31 \mathrm{~V}$ and cathodic peak at $0.27 \mathrm{~V}$ ) (Figure 3B). This indicated that the oxidation of the doped network required a lower potential compared to the undoped network. The CVs of the undoped system also showed a quasi-reversible process with oxidation and reduction peak separation increasing with potential scan rate. Following doping, the CVs became more reversible (Figure 3B), which indicated a faster charge transport kinetics due to the higher electrical conductivity in the doped system.

We could also infer from the linear relation between the potential scan rate and anodic peak current that both the doped and undoped hydrogels were in good contact with the electrode surface (Figure S9). In contrast, PAA hydrogels displayed no redox activity as shown by the absence of oxidation/reduction waves in their cyclic voltammogram (Figure S10). The redox activity of the doped hydrogels was further confirmed by electrochemical impedance spectroscopy (EIS) conducted at $290 \mathrm{mV}$ (Figure S11). Unlike the Nyquist plot of the PAA system, the Nyquist plot of the doped hydrogel showed a quasisemicircular arc in the high frequency regime (inset Figure S11). This implies a redox activity as opposed to a capacitive behavior of the nonredox active PAA system. This was further evidence that the modified PEDOT scaffold retained its electroactive properties in aqueous electrolytes and despite the attachment of functional groups to its side chains.

Envisioned applications of the PEDOT-COOH as a component of bioelectronic devices will require the polymer to be in contact with electroresponsive tissue. Additionally, the 

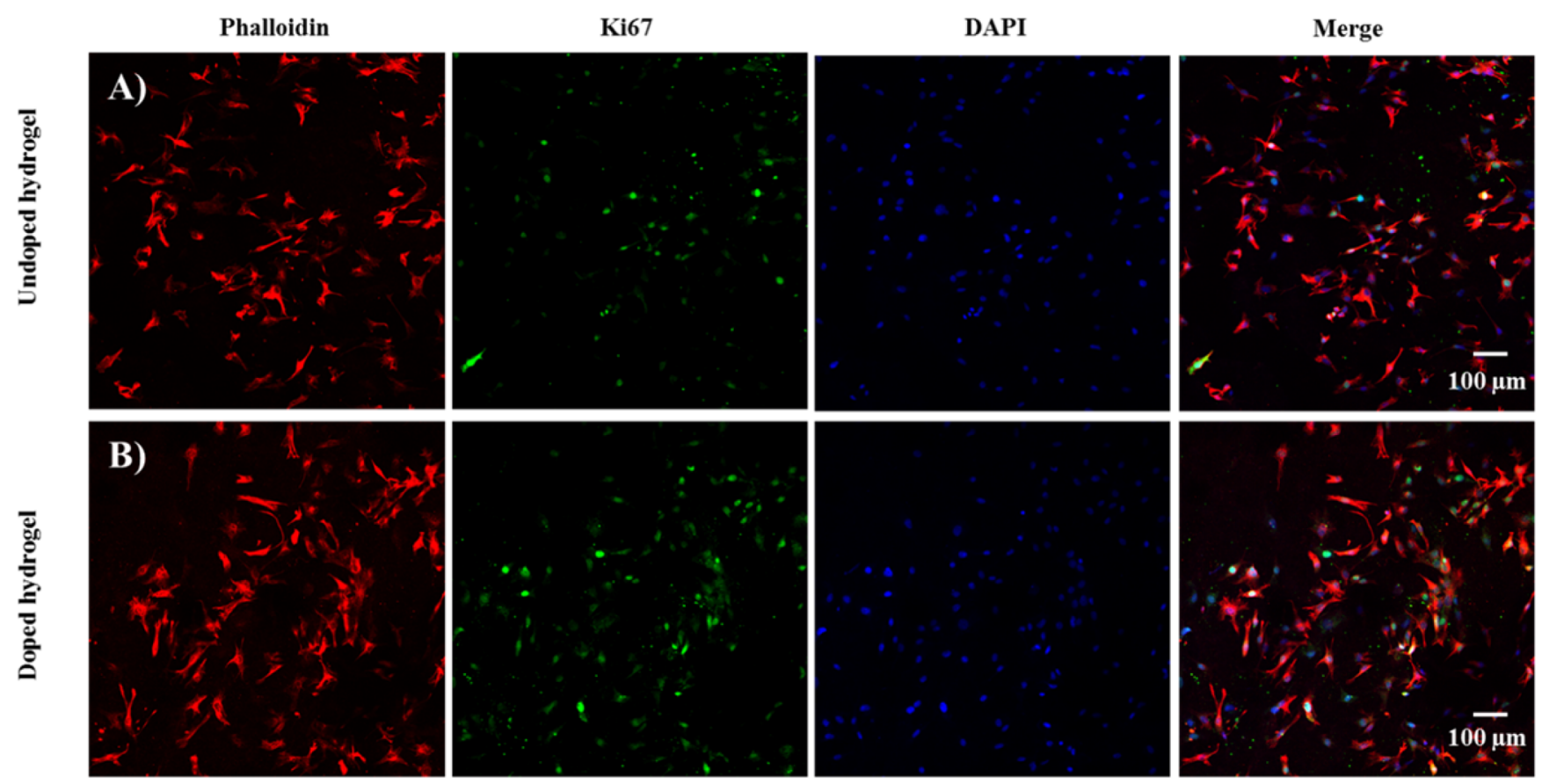

Undoped Hydrogel

Day 3

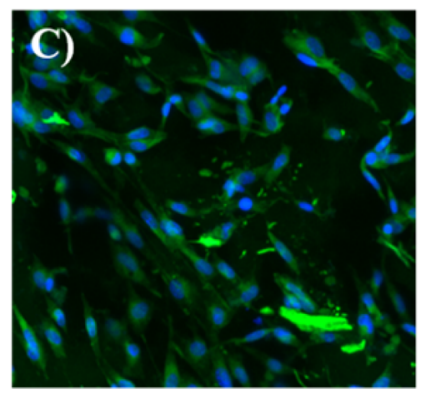

Day 7

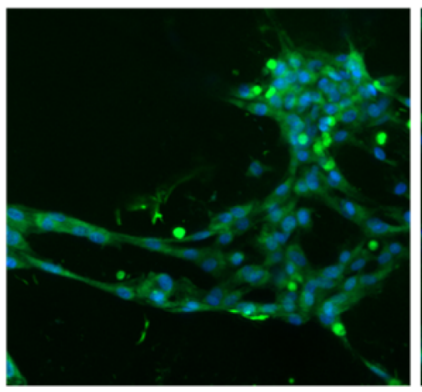

Doped Hydrogel

Day 3

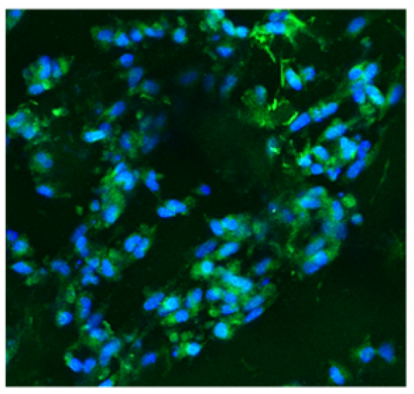

Day 7

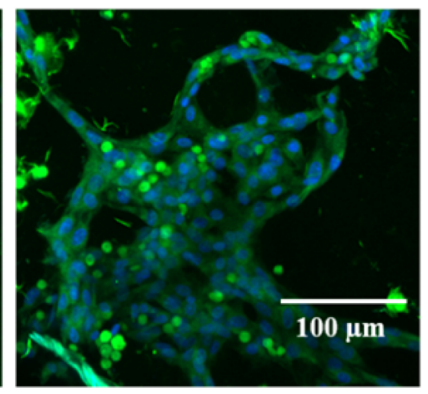

Figure 4. $\mathrm{C} 2 \mathrm{C} 12$ cultured in f-PEDOT hydrogel; proliferation of cells in undoped (A) and doped hydrogels (B) at day 3. Proliferation is indicated by positive nuclear Ki67 staining (green), while phalloidin staining for actin (red) allows visualization of the cell shape. DAPI stain labels the DNA in nuclei (blue). C) Staining for the mature muscle marker myosin heavy chain IIb (MHC IIb) (green) and DAPI (blue) in undoped and doped hydrogel at day 3 and day 7. The presence of MHCIIb shows that the C2C12s, which are immature muscle cells and normally have low expression of MHCIIb, are becoming mature muscle cells when seeded on the hydrogel. While the C2C12s exist as single cells at day 3, multicellular tubes are observed by day 7 showing increased maturation with continued culture time.

conductive and electroactive nature of our developed f-PEDOT hydrogel in the swollen state, along with its favorable swelling ratio and mechanical integrity, make it suitable as a $3 \mathrm{D}$-scaffold for the proliferation and differentiation of electro-responsive cells. C2C12 cells are a mouse myoblast cell line commonly used as an in vitro model to study muscle differentiation. We seeded $\mathrm{C} 2 \mathrm{C} 12$ cells on both undoped and doped scaffolds and assessed qualitatively for adhesion and viability at day 3. As shown in Figure S12, the f-PEDOT hydrogels promoted cell adhesion without the need to precoat the scaffold with any celladhesion protein (such as fibronectin or laminin). Significantly, other conjugated based systems normally require modification of their surfaces with adhesive molecules to enhance their cytocompatibility and cell attachment. Of particular interest was the migration of the cells from the surface into the $3 \mathrm{D}$ highly swollen and porous structure of the hydrogels (Figure S13). We found that cells penetrated to a depth of $\sim 2 \mathrm{~mm}$, which confirmed the cytocompatibility of the conductive hydrogel
(Figure S13E-G). The structural composition of the $\mathrm{C} 2 \mathrm{C} 12$ cells was confirmed by phalloidin stain for actin (Figure 4A,B). Actin is a critical component in many structural and functional processes in the cell. The phalloidin staining confirmed that the structural network remained undamaged on both doped and undoped hydrogels. Proliferation of C2C12 cells was confirmed by Ki67 staining on both undoped and doped hydrogels (Figure 4A,B). The Ki67 protein is present in the cell nucleus during all active phases of the cell cycle (G1, S, G2, and mitosis) but is absent from resting cells (G0). Detection of the Ki67 protein by immunostaining indicates that the cells are proliferating; thus, the hydrogels support muscle cell expansion. When $\mathrm{C} 2 \mathrm{C} 12$ myoblasts, immature muscle cells, are grown to a high density, they can begin to differentiate. To facilitate this process, the cells are placed in low serum conditions as the growth factor withdrawal initiates a differentiation program. In this multistep process, the myoblasts stop proliferating, elongate, and align next to each other before they are then in 
a position to fuse together to form multinucleated tubes capable of contraction. Cells grown in the hydrogel were observed to align at day 3 (Figures 4C and S12 and S14), and multinucleated tubes were present by day 7 (Figure 4C) indicating that cells were able to differentiate in the hydrogels. In addition to morphological changes, maturing muscle myoblasts such as $\mathrm{C} 2 \mathrm{C} 12 \mathrm{~s}$ begin to produce proteins required for their contractile function, such as MHC IIb (Figure 4C). $\mathrm{MHC} \mathrm{IIb}$ is a motor protein that generates force to drive muscle contraction and is an essential component of functional muscle. Expression of this protein is therefore used as a marker of $\mathrm{C} 2 \mathrm{C} 12$ maturation. The presence of $\mathrm{MHC} \mathrm{IIb}$ in cells grown in both doped and undoped hydrogels shows that both forms of the hydrogel are conducive to the maturation of muscle cells.

In summary, we found that $\mathrm{C} 2 \mathrm{C} 12$ cells grown in both the doped and undoped samples formed myotube structures and expressed MHC IIb (Figure 4C) indicating that they were differentiating. Coupled with their expression of Ki67 (Figure $4 A, B)$, this showed that our hydrogels were capable of sustaining the differentiation of $\mathrm{C} 2 \mathrm{C} 12$ cells in addition to their proliferating precursors. This in vitro study was conducted as evidence of biocompatibility of the newly fabricated PEDOT based hydrogels. Future studies will investigate the mechanistic insight into the role of the hydrogel on cellular differentiation.

\section{CONCLUSION}

In this study, we successfully produced an electroactive hydrogel network composed of PEDOT covalently bound to a hydrophilic polymer. This led to the formation of a scaffold combining both the appropriate physical properties of a hydrogel and the electronic characteristics of a conjugated polymer. We achieved this by synthesizing a processable PEDOT with functional groups tethered on the side chains that could be further modified to fabricate a covalently linked polymeric network. The PEDOT-COOH was shown to be electroactive as both a pristine polymer and a component of the hydrogel. Finally, we demonstrated the suitability of these scaffolds as hydrated 3D structures for cell adhesion, proliferation, and differentiation. The next step will be to employ these electroactive hydrogels in bioelectronics devices and test their capacity to either electrically stimulate electroresponsive cells or probe the bioelectric signals. Covalently linking PEDOT with hydrophilic polymers to produce scaffolds of tailored properties represents a major advance in tissue engineering that paves the way for the smart design of conductive functional scaffolds.

\section{ASSOCIATED CONTENT}

\section{S Supporting Information}

The Supporting Information is available free of charge on the ACS Publications website at DOI: 10.1021/acs.chemmater.6b01298.

Tables of hydrogel dimensions and elemental analysis; ${ }^{1} \mathrm{H}$-NMR data, solid state CP-MAS ${ }^{13} \mathrm{C}$ data, UV-vis absorption spectra, redox peak currents, percentage swelling ratios, cyclic voltammograms, Nyquist plot, images of $\mathrm{C} 2 \mathrm{C} 12$ cultures and f-PEDOT hydrogel (PDF)

\section{AUTHOR INFORMATION}

\section{Corresponding Authors}

*E-mail: Damia.mawad@unsw.edu.au.
*E-mail: m.stevens@imperial.ac.uk.

\section{Author Contributions}

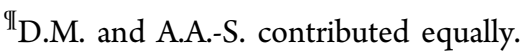

\section{Funding}

D.M. was supported by Marie Curie actions FP7 through the Intra-European Marie Curie Fellowship "MultiFun CP” under grant agreement no. 328897. M.M.S. acknowledges the support from the ERC Seventh Framework Programme Consolidator grant "Naturale CG" under grant agreement no. 616417 and a Wellcome Trust Senior Investigator Award (098411/Z/12/Z) for funding. M.M.M. would like to thank the Public Service Department of Malaysia for the $\mathrm{PhD}$ scholarship via King of Malaysia scheme and University Teknologi MARA (UiTM). J.T. acknowledges the British Heart Foundation grant RM/13/ $1 / 30157$.

\section{Notes}

The authors declare no competing financial interest.

\section{ACKNOWLEDGMENTS}

The authors would like to acknowledge Dr. Douglas Lawes and Dr. James Hook of the NMR Facility within the Mark Wainwright Analytical Centre at the University of New South Wales for NMR support. Also, we would like to thank Dr. Patricia Y. Hayes from the Australian National Fabrication Facility (ANFF) and Dr. Pawel Wagner from the ARC Centre of Excellence for Electromaterials Science (ACES) for conducting the GPC experiment.

\section{ABBREVIATIONS}

AA, acrylic acid; AIBN, 2,2'-azobis(2-methyl-proprionitrile); APMA, $N$-(3-aminopropyl) methacrylamide hydrochloride; $\mathrm{CP}$, conducting polymer; DMEM, Dulbecco's modified Eagle's medium; EDC, $N$-(3-(dimethylamino)propyl)- $N^{\prime}$-ethylcarbodiimide hydrochloride; HOMO, highest occupied molecular orbital; LUMO, lowest unoccupied molecular orbital; PAA, poly(acrylic acid); PEDOT, poly(ethylenedioxy thiophene); PEG-DA, poly(ethylene glycol) diacrylate; TBAB, tetrabutylammonium borate

\section{REFERENCES}

(1) Liao, C.; Zhang, M.; Yao, M. Y.; Hua, T.; Li, L.; Yan, F. Flexible Organic Electronics in Biology: Materials and Devices. Adv. Mater. 2015, 27, 7493-7527.

(2) Owens, R. M.; Malliaras, G. G. Organic Electronics at the Interface with Biology. MRS Bull. 2010, 35, 449-456.

(3) Stavrinidou, E.; Gabrielsson, R.; Gomez, E.; Crispin, X.; Nilsson, O.; Simon, D. T.; Berggren, M. Electronic Plants. Sci. Adv. 2015, 1, e1501136.

(4) Gkoupidenis, P.; Schaefer, N.; Garlan, B.; Malliaras, G. G. Neuromorphic Functions in PEDOT: PSS Organic Electrochemical Transistors. Adv. Mater. 2015, 27, 7176-7180.

(5) Zhu, B.; Luo, S.-C.; Zhao, H.; Lin, H.-A.; Sekine, J.; Nakao, A.; Chen, C.; Yamashita, Y.; Yu, H. Large Enhancement in Neurite Outgrowth on a Cell Membrane-Mimicking Conducting Polymer. Nat. Commun. 2014, 5, 4523.

(6) Wang, L.; Feng, F.; Ma, Z. Novel Electrochemical Redox-active Species: One-Step Synthesis of Polyaniline Derivative-Au/Pd and Its Application for Multiplexed Immunoassay. Sci. Rep. 2015, 5, 16855.

(7) Mawad, D.; Molino, P.; Gambhir, S.; Locke, J.; Officer, D.; Wallace, G. Electrically Induced Disassembly of Electroactive Multilayer Films Fabricated from Water Soluble Polythiophenes. Adv. Funct. Mater. 2012, 22, 5020-5027. 
(8) Mawad, D.; Gilmore, K.; Molino, P.; Wagner, K.; Wagner, P.; Officer, D.; Wallace, G. An Erodible Polythiophene-Based Composite for Biomedical Applications. J. Mater. Chem. 2011, 21, 5555-5560.

(9) Seyedin, M. Z.; Razal, J. M.; Innis, P. C.; Wallace, G. G. StrainResponsive Polyurethane/PEDOT:PSS Elastomeric Composite Fibers with High Electrical Conductivity. Adv. Funct. Mater. 2014, 24, 29572966.

(10) Lu, Y.; He, W.; Cao, T.; Guo, H.; Zhang, Y.; Li, Q.; Shao, Z.; Cui, Y.; Zhang, X. Elastic, Conductive, Polymeric Hydrogels and Sponges. Sci. Rep. 2014, 4, 5792.

(11) Balint, R.; Cassidy, N. J.; Cartmell, S. H. Conductive Polymers: Towards a Smart Biomaterial for Tissue Engineering. Acta Biomater. 2014, 10, 2341-2353.

(12) Ding, H.; Zhong, M.; Kim, Y. J.; Pholpabu, P.; Balasubramanian, A.; Hui, C. M.; He, H.; Yang, H.; Matyjaszewski, K.; Bettinger, C. J. Biologically Derived Soft Conducting Hydrogels Using HeparinDoped Polymer Networks. ACS Nano 2014, 8, 4348-4357.

(13) Aldissl, M. Chain Rigidity-Processability Correlation in Inherently Conducting Polymers. Polym.-Plast. Technol. Eng. 1987, 26, 45-70.

(14) Abidian, M. R.; Corey, J. M.; Kipke, D. R.; Martin, D. C. Conducting-Polymer Nanotubes Improve Electrical Properties, Mechanical Adhesion, Neural Attachment, and Neurite Outgrowth of Neural Electrodes. Small 2010, 6, 421-429.

(15) MacLean, M. W. A.; Kitao, T.; Suga, T.; Mizuno, M.; Seki, S.; Uemura, T.; Kitagawa, S. Unraveling Inter-and Intrachain Electronics in Polythiophene Assemblies Mediated by Coordination Nanospaces. Angew. Chem., Int. Ed. 2016, 55, 708-713.

(16) Mawad, D.; Boughton, E. A.; Boughton, P.; Lauto, A. Advances in Hydrogels Applied to Degenerative Diseases. Curr. Pharm. Des. 2012, 18, 2558-2575.

(17) Annabi, N.; Tamayol, A.; Uquillas, J. A.; Akbari, M.; Bertassoni, L. E.; Cha, C.; Camci-Unal, G.; Dokmeci, M. R.; Peppas, N. A.; Khademhosseini, A. 25th Anniversary Article: Rational design and Applications of Hydrogels in Regenerative Medicine. Adv. Mater. 2014, 26, 85-123.

(18) Runge, M. B.; Dadsetan, M.; Baltrusaitis, J.; Ruesink, T.; Lu, L.; Windebank, A. J.; Yaszemski, M. J. Development of Electrically Conductive Oligo(polyethylene glycol) Fumarate-Polypyrrole Hydrogels for Nerve Regeneration. Biomacromolecules 2010, 11, 2845-2853.

(19) Sirivisoot, S.; Pareta, R.; Harrison, B. S. Protocol and Cell Responses in Three Dimensional Conductive Collagen Gel Scaffolds with Conductive Polymer Nanofibres for Tissue Regeneration. Interface Focus 2014, 4, 20130050.

(20) Mawad, D.; Stewart, E.; Officer, D. L.; Romeo, T.; Wagner, P.; Wagner, K.; Wallace, G. G. A Single Component Conducting Polymer Hydrogel as a Scaffold for Tissue Engineering. Adv. Funct. Mater. 2012, 22, 2692-2699.

(21) Du, R.; Xu, Y.; Luo, Y.; Zhang, X.; Zhang, J. Synthesis of Conducting Polymer Hydrogels with 2D Building Blocks and Their Potential-dependent Gel-sol Transitions. Chem. Commun. 2011, 47, 6287-6289.

(22) Lin, J.; Tang, Q.; Wu, J.; Li, Q. A Multifunctional Hydrogel with High-conductivity, pH-responsive, and Release Properties from Polyacrylate/Polypyrrole. J. Appl. Polym. Sci. 2010, 116, 1376-1383.

(23) Tang, Q.; Wu, J.; Sun, H.; Lin, J.; Fan, S.; Hu, D. Polyaniline/ Polyacrylamide Conducting Composite Hydrogel with a Porous Structure. Carbohydr. Polym. 2008, 74, 215-219.

(24) Wei, D.; Lin, X.; Li, L.; Shang, S.; Yuen, M. C.; Yan, G.; Yu, X. Controlled Growth of Polypyrrole Hydrogels. Soft Matter 2013, 9, 2832-2836.

(25) Antonio, J.; Lira, L. M.; Goncales, V. R.; Cordoba de Torresi, S. I. Fully Conducting Hydro-sponges with Electro-swelling Properties Tuned by Synthetic Parameters. Electrochim. Acta 2013, 101, 216224.

(26) Wan, A. M-D.; Inal, S.; Williams, T.; Wang, K.; Leleux, P.; Estevez, L.; Giannelis, E. P.; Fischbach, C.; Malliaras, G. G.; Gourdon, D. 3D Conducting Polymer Platforms for Electrical Control of Protein
Conformation and Cellular Functions. J. Mater. Chem. B 2015, 3, 5040-5048.

(27) Chakraborty, P.; Bairi, P.; Mondal, S.; Nandi, A. K. CoAssembled Conductive Hydrogel of N-Fluorenylmethoxycarbonyl Phenylalanine with Polyaniline. J. Phys. Chem. B 2014, 118, 1396913980.

(28) Pan, L.; Yu, G.; Zhai, D.; Lee, H. R.; Zhao, W.; Liu, N.; Wang, H.; Tee, B. C.; Shi, Y.; Cui, Y.; Bao, Z. Hierarchical Nanostructured Conducting Polymer Hydrogel with High Electrochemical Activity. Proc. Natl. Acad. Sci. U. S. A. 2012, 109, 9287-9292.

(29) Xie, H.; Luo, S. C.; Yu, H. Electric-field-assisted Growth of Functionalized Poly(3,4-ethylenedioxythiophene) Nanowires for Label-free Protein Detection. Small 2009, 5, 2611-2617.

(30) Groenendaal, L. B.; Jonas, F.; Freitag, D.; Pielartzik, H.; Reynolds, J. R. Poly(3,4-ethylenedioxythiophene) and Its Derivatives: Past, Present, and Future. Adv. Mater. 2000, 12, 481-494.

(31) Roncali, J.; Blanchard, P.; Frere, P. 3,4-Ethylenedioxythiophene (EDOT) as a Versatile Building Block for Advanced Functional $\pi$ Conjugated Systems. J. Mater. Chem. 2005, 15, 1589-1610.

(32) Schottland, P.; Fichet, O.; Teyssie, D.; Chevrot, C. LangmuirBlodgett Films of an Alkoxy Derivative of Poly(3,4-ethylenedioxythiophene). Synth. Met. 1999, 101, 7-8.

(33) Luo, S.; Ali, E. M.; Tansil, N. C.; Yu, H.; Gao, S.; Kantchev, E.; Ying, J. Y. Poly(3,4-ethylenedioxythiophene) (PEDOT) Nanobiointerfaces: Thin, Ultrasmooth, and Functionalized PEDOT Films with in Vitro and in Vivo Biocompatibility. Langmuir 2008, 24, 8071-8077.

(34) Lee, S.; Gleason, K. K. Enhanced Optical Property with Tunable Band Gap of Cross-linked PEDOT Copolymers via Oxidative Chemical Vapor Deposition. Adv. Funct. Mater. 2015, 25, 85-93.

(35) Zhang, Q.; Feng, J.; Liu, K.; Zhu, D.; Yang, M.; Ye, H.; Liu, X. Synthesis and Characterization of Novel Low Band Gap Polymers: Poly(heteroarylene methines). Synth. Met. 2006, 156, 804-808.

(36) İçli, M.; Pamuk, M.; Algi, F.; Önal, A. M.; Cihaner, A. DonorAcceptor Polymer Electrochromes with Tunable Colors and Performance. Chem. Mater. 2010, 22, 4034-4044.

(37) Patra, A.; Wijsboom, Y. H.; Zade, S. S.; Li, M.; Sheynin, Y.; Leitus, G.; Bendikov, M. Poly(3,4-ethylenedithioselenophene). J. Am. Chem. Soc. 2008, 130, 6734-6736.

(38) Chen, W.; Xue, G. Low Potential Electrochemical Syntheses of Heteroaromatic Conducting Polymers in a Novel Solvent System Based on Trifluroborate-Ethyl Ether. Prog. Polym. Sci. 2005, 30, 783811.

(39) Ming, S.; Zhen, S.; Lin, K.; Zhao, L.; Xu, J.; Lu, B. Thiadiazolo[3,4-c]pyridine as an Acceptor toward Fast-Switching Green Donor-Acceptor-Type Electrochromic Polymer with Low Bandgap. ACS Appl. Mater. Interfaces 2015, 7, 11089-11098.

(40) Lago, M. A.; Grinberg, V. Y.; Burova, T. V.; Concheiro, A.; Alvarez-Lorenzo, C. Ionic and Polyampholyte $N$-IsopropylacrylamideBased Hydrogels Prepared in the Presence of Imprinting Ligands: Stimuli-Responsiveness and Adsorption/Release Properties. J. Funct. Biomater. 2011, 2, 373-390.

(41) Khandaker, M. Poly(Vinyl Alcohol)/Poly(Acrylic Acid) Hydrogel in a dc Electric Field Swelling, Shape Change, and Actuation Characteristics. Int. J. Mater. Sci. 2013, 3, 133-138.

(42) Engler, A. J.; Sen, S.; Sweeney, H. L.; Discher, D. E. Matrix Elasticity Directs Stem Cell Lineage Specification. Cell 2006, 126, 677-689. 